\title{
Properties of Expected Residual Minimization Model for a Class of Stochastic Complementarity Problems
}

\author{
Mei-Ju Luo ${ }^{1}$ and Yuan Lu' \\ ${ }^{1}$ School of Mathematics, Liaoning University, Liaoning 110036, China \\ ${ }^{2}$ School of Sciences, Shenyang University, Liaoning 110044, China \\ Correspondence should be addressed to Mei-Ju Luo; meiju_luo@yahoo.cn \\ Received 31 January 2013; Revised 10 May 2013; Accepted 14 May 2013 \\ Academic Editor: Farhad Hosseinzadeh Lotfi
}

Copyright (C) 2013 M.-J. Luo and Y. Lu. This is an open access article distributed under the Creative Commons Attribution License, which permits unrestricted use, distribution, and reproduction in any medium, provided the original work is properly cited.

\begin{abstract}
Expected residual minimization (ERM) model which minimizes an expected residual function defined by an NCP function has been studied in the literature for solving stochastic complementarity problems. In this paper, we first give the definitions of stochastic $P$-function, stochastic $P_{0}$-function, and stochastic uniformly $P$-function. Furthermore, the conditions such that the function is a stochastic $P\left(P_{0}\right)$-function are considered. We then study the boundedness of solution set and global error bounds of the expected residual functions defined by the "Fischer-Burmeister" (FB) function and "min" function. The conclusion indicates that solutions of the ERM model are robust in the sense that they may have a minimum sensitivity with respect to random parameter variations in stochastic complementarity problems. On the other hand, we employ quasi-Monte Carlo methods and derivative-free methods to solve ERM model.
\end{abstract}

\section{Introduction}

Given a vector-valued function $F: \mathbf{R}^{n} \times \Omega \rightarrow \mathbf{R}^{n}$, the stochastic complementarity problems, denoted by $\operatorname{SCP}(F(x, \omega))$, are to find a vector $x^{*}$ such that

$$
\begin{gathered}
x^{*} \geq 0, \quad F\left(x^{*}, \omega\right) \geq 0, \\
\left(x^{*}\right)^{T} F\left(x^{*}, \omega\right)=0, \quad \omega \in \Omega \text { a.s., }
\end{gathered}
$$

where $\omega \in \Omega \subseteq \mathbf{R}^{m}$ is a random vector with given probability distribution $\mathscr{P}$ and "a.s." means "almost surely" under the given probability measure. Particularly, when $F$ is an affine function of $x$ for any $\omega$, that is,

$$
F(x, \omega)=M(\omega) x+q(\omega), \quad \omega \in \Omega,
$$

where $M(\omega) \in \mathbf{R}^{n \times n}$ and $q(\omega) \in \mathbf{R}^{n}$, the $\operatorname{SCP}(F(x, \omega))$ is called stochastic linear complementarity problems, denoted by $\operatorname{SLCP}(M(\omega), q(\omega))$. Correspondingly, problem (1) is called stochastic nonlinear complementarity problem, denoted by $\operatorname{SNCP}(F(x, \omega))$ if $F$ can not be denoted by an affine function of $x$ for any $\omega$. The deterministic problems, which are called complementarity problems (denoted by $\mathrm{CP}(F(x))$ ), have been intensively studied. More information about theoretical analysis, numerical algorithms and applications especially in economics and engineering can be found in comprehensive books $[1,2]$.

In practical applications, some elements may involve stochastic factors. In fact, due to stochastic factors, the function value of $F$ depends not only on $x$, but also on random vectors. Hence, problem (1) does not have solution in general for almost all $\omega \in \Omega$. To solve these problems, researchers focus on giving reasonable deterministic reformulations for $\operatorname{SCP}(F(x, \omega))$. Certainly, different deterministic formulations may yield different solutions that are optimal in different senses. In the study of $\operatorname{SCP}(F(x, \omega))$, three types of formulations have been proposed; the expected value (EV) formulation, the expected residual minimization (ERM) formulation, and the SMPEC formulation [3].

The EV formulation is studied by Gürkan et al. [4]. The problem considered in [4] is actually a stochastic variational inequality problem. When applied to the $\operatorname{SCP}(F(x, \omega))$, the $\mathrm{EV}$ model can be stated as follows:

$$
x^{*} \geq 0, \quad \mathbf{E}\left[F\left(x^{*}, \omega\right)\right] \geq 0, \quad\left(x^{*}\right)^{T} \mathbf{E}\left[F\left(x^{*}, \omega\right)\right]=0,
$$

where $\mathbf{E}$ means expectation with respect to $\omega$. 
The ERM model is first proposed by Chen and Fukushima [5] for solving the $\operatorname{SLCP}(M(\omega), q(\omega))$. By employing an NCP function $\phi$, the $\operatorname{SCP}(F(x, \omega))(1)$ is transformed equivalently to the stochastic equations

$$
\Phi(x, \omega)=0, \quad \omega \in \Omega \text { a.s. }
$$

where $\Phi: \mathbf{R}^{n} \times \Omega \rightarrow \mathbf{R}^{n}$ is defined by

$$
\Phi(x, \omega):=\left(\begin{array}{c}
\phi\left(x_{1}, F_{1}(x, \omega)\right) \\
\vdots \\
\phi\left(x_{n}, F_{n}(x, \omega)\right)
\end{array}\right),
$$

and $x_{i}$ denotes the $i$ th component of the vector $x$. Here $\phi$ : $\mathbf{R}^{n} \rightarrow \mathbf{R}$ is an NCP function which has the property

$$
\phi(a, b)=0 \Longleftrightarrow a \geq 0, \quad b \geq 0, a b=0 .
$$

Then the ERM formulation for (1) is given by

$$
\min _{x \in \mathbf{R}_{+}^{n}} \theta(x):=\mathbf{E}\left[\|\Phi(x, \omega)\|^{2}\right] .
$$

The NCP functions employed in [5] include the FischerBurmeister function, which is defined by

$$
\phi_{\mathrm{FB}}(a, b):=\sqrt{a^{2}+b^{2}}-(a+b)
$$

and the min function

$$
\phi_{\min }(a, b):=\min \{a, b\} .
$$

In particular, it is known $[6,7]$ that there exist the following relations between these two functions:

$$
\frac{2}{\sqrt{2}+2}\left|\phi_{\min }\right| \leq\left|\phi_{\mathrm{FB}}\right| \leq(\sqrt{2}+2)\left|\phi_{\min }\right| \text {. }
$$

As observed in [5], the ERM formulations with different NCP functions may have different properties. Subsequently, the ERM formulation for $\operatorname{SCP}(F(x, \omega))$ has been studied in $[6,8-13]$. Note that Fang et al. [8] propose a new concept of stochastic matrice: $M(\cdot)$ is called a stochastic $R_{0}$ matrix if

$$
\mathscr{P}\left\{\omega: x \geq 0, M(\omega) x \geq 0, x^{T} M(\omega) x=0\right\}=1 \Longrightarrow x=0 .
$$

Moreover, Zhang and Chen [11] introduce a new concept of stochastic $R_{0}$ function, which can be regarded as a generalization of the stochastic $R_{0}$ matrix given in [8].

Throughout this paper, we suppose that the sample space $\Omega$ is nonempty and compact set and that the function $F(x, \omega)$ is continuous with respect to $x$ and $\omega$. On the other hand, we will use the following notations: $I(x)=\left\{i: x_{i}=0\right\}$ and $J(x)=\left\{i: x_{i} \neq 0\right\}$ for a given vector $x \in \mathbf{R}^{n} .\langle l, n\rangle$ represents the set $\{l, l+1, \ldots, l+n\}$ for natural numbers $l$ and $u$ with $l<u . x_{+}=\max \{x, 0\}$ for any given vector $x .\|\cdot\|$ refers to the Euclidean norm.

The remainder of the paper is organized as follows: in Section 2, we introduce the concepts of a stochastic $P$ function, a stochastic $P_{0}$-function, and a stochastic uniformly
$P$-function, which can be regarded as a generalization of the deterministic $P, P_{0}$-function, and uniformly $P$-function or an extension of stochastic $P$ matrix and stochastic $P_{0}$ matrix [14]. In addition, some properties of a stochastic $P\left(P_{0}\right)$-function are given. In Section 3, we show the sufficient conditions for the solution set of ERM problem to be nonempty and bounded. In Section 4, we discuss error bounds of $\operatorname{SCP}(F(x, \omega))$. In Section 5 , an algorithm will be given to solve ERM model. We then give conclusions in Section 6.

\section{Stochastic $P\left(P_{0}\right)$-Function}

It is well known that the $P$-function, $P_{0}$-function, and uniformly $P$-function play an important role in the nonlinear complementarity problems theory [1]. We will introduce a new concept of stochastic $P$-function, $P_{0}$-function, and uniformly $P$-function, which can be regarded as a generalization of their deterministic form or stochastic $P$ matrix and stochastic $P_{0}$ matrix.

Definition 1 (see [14]). $M(\cdot)$ is called a stochastic $P\left(P_{0}\right)$-matrix if there exists $i \in J(x)$ such that, for every $x \neq 0$ in $\mathbf{R}^{n}$,

$$
\mathscr{P}\left\{\omega: x_{i}(M(\omega) x)_{i}>0(\geq 0)\right\}>0
$$

Definition 2. A function $F: \mathbf{R}^{n} \times \Omega \rightarrow \mathbf{R}^{n}$ is a stochastic $P\left(P_{0}\right)$-function if there exist $i \in J(x, y), i \in\langle 1, n\rangle$ such that, for every $x \neq y$ in $\mathbf{R}^{n}$,

$$
\begin{gathered}
x_{i} \neq y_{i}, \\
\mathscr{P}\left\{\omega:\left(x_{i}-y_{i}\right)\left(F_{i}(x, \omega)-F_{i}(y, \omega)\right)>0(\geq 0)\right\}>0 .
\end{gathered}
$$

Definition 3. A function $F: \mathbf{R}^{n} \times \Omega \rightarrow \mathbf{R}^{n}$ is a stochastic uniformly $P$-function if there exists a positive constant $\alpha$ and $i \in J(x, y), i \in\langle 1, n\rangle$ such that, for every $x \neq y$ in $\mathbf{R}^{n}$,

$$
\mathscr{P}\left\{\omega:\left(x_{i}-y_{i}\right)\left(F_{i}(x, \omega)-F_{i}(y, \omega)\right) \geq \alpha\|x-y\|^{2}\right\}>0 .
$$

Clearly, every stochastic uniformly $P$-function must be a stochastic $P$-function, which in turn must be a stochastic $P_{0^{-}}$ function. We further cite the definition of "equicoercive" in [11]. More information about this definition can be found in $[11]$.

Definition 4 (see [11]). We say that $F: \mathbf{R}^{n} \times \Omega \rightarrow \mathbf{R}^{n}$ is equicoercive on $\mathscr{D} \subseteq \mathbf{R}^{n}$, if, for any $\left\{x^{k}\right\} \subseteq \mathscr{D}$ satisfying $\left\|x^{k}\right\| \rightarrow \infty$, the existence of $\left\{\omega^{k}\right\} \subseteq \operatorname{supp} \Omega$ with $\lim _{k \rightarrow \infty} F_{i}\left(x^{k}, \omega^{k}\right)=\infty\left(\lim _{k \rightarrow \infty}\left(-F_{i}\left(x^{k}, \omega^{k}\right)\right)_{+}=\infty\right)$ for some $i \in\langle 1, n\rangle$ implies that

$$
\begin{aligned}
\mathscr{P}\{\omega & \left.: \lim _{k \rightarrow \infty} F_{i}\left(x^{k}, \omega\right)=\infty\right\} \\
& >0\left(\mathscr{P}\left\{\omega: \lim _{k \rightarrow \infty}\left(-F_{i}\left(x^{k}, \omega\right)\right)_{+}=\infty\right\}>0\right),
\end{aligned}
$$


where

$\operatorname{supp} \Omega$

$$
:=\left\{\bar{\omega} \in \Omega: \int_{B_{\omega}(\bar{\omega}, v) \cap \Omega} d F(\omega)>0 \text { for any } \nu>0\right\}
$$

and $B_{\omega}(\bar{\omega}, \nu):=\{\omega:\|\omega-\bar{\omega}\|<\nu\}$ and $F(\omega)$ is the distribution function of $\omega$.

More details about supp $\Omega$ were included in [8].

Proposition 5. If $F$ is a stochastic $P_{0}$-function, then $F+\varepsilon x$ is a stochastic $P$-function for every $\varepsilon>0$.

Proof. From the definition of stochastic $P_{0}$-function, there exist $i \in J(x, y), i \in\langle 1, n\rangle$ such that, for every $x \neq y$,

$$
x_{i} \neq y_{i}, \quad \mathscr{P}\left\{\omega:\left(x_{i}-y_{i}\right)\left(F_{i}(x, \omega)-F_{i}(y, \omega)\right) \geq 0\right\}>0 .
$$

Hence, we have

$$
\begin{aligned}
\mathscr{P} & \left\{\omega:\left(x_{i}-y_{i}\right)\left(F_{i}(x, \omega)+\varepsilon x_{i}-\left(F_{i}(y, \omega)+\varepsilon y_{i}\right)\right)>0\right\} \\
& =\mathscr{P}\left\{\omega:\left(x_{i}-y_{i}\right)\left(F_{i}(x, \omega)-F_{i}(y, \omega)\right)+\varepsilon\left(x_{i}-y_{i}\right)^{2}>0\right\} \\
& \geq \mathscr{P}\left\{\omega:\left(x_{i}-y_{i}\right)\left(F_{i}(x, \omega)-F_{i}(y, \omega)\right) \geq 0\right\}>0 .
\end{aligned}
$$

This proposition gives the relationship between stochastic $P_{0}$-function and stochastic $P$-function.

Proposition 6. Let $F$ be an affine function of $x$ for any $\omega \in \Omega$ defined by (2). Then $F$ is a stochastic $P\left(P_{0}\right)$-function if and only if $M(\cdot)$ is a stochastic $P\left(P_{0}\right)$ matrix.

Proof. By the definition of stochastic $P\left(P_{0}\right)$-function, we have that there exist $i \in J(x, y), i \in\langle 1, n\rangle$ such that, for every $x \neq y$,

$$
\begin{gathered}
x_{i} \neq y_{i}, \\
\mathscr{P}\left\{\omega:\left(x_{i}-y_{i}\right)\left(F_{i}(x, \omega)-F_{i}(y, \omega)\right)>0(\geq 0)\right\}>0,
\end{gathered}
$$

which is equivalent to

$$
\begin{gathered}
x_{i} \neq y_{i}, \\
\mathscr{P}\left\{\omega:\left(x_{i}-y_{i}\right)(M(\omega)(x-y))_{i}>0(\geq 0)\right\}>0,
\end{gathered}
$$

when $F$ is defined by (2). Set $z=x-y$; then $z \neq 0$, and we have that formulation (20) holds if and only if there exists $i \in J(z)$ such that, for every $z \neq 0$,

$$
\mathscr{P}\left\{\omega: z_{i}(M(\omega) z)_{i}>0(\geq 0)\right\}>0 .
$$

Hence, $M(\cdot)$ is a stochastic $P\left(P_{0}\right)$ matrix.

Proposition 7. $F$ is a stochastic $P\left(P_{0}\right)$-function if and only if there exists a $\bar{\omega} \in \operatorname{supp} \Omega$ such that $F(\cdot, \bar{\omega})$ is a $P\left(P_{0}\right)$-function.
Proof. For the "if" part, suppose on the contrary that $F$ is not a stochastic $P\left(P_{0}\right)$-function, and then there exist $\tilde{x}, \tilde{y}, \tilde{x} \neq \tilde{y}$ in $\mathbf{R}^{n}$ for any $i \in\langle 1, n\rangle$ satisfying

$$
\begin{gathered}
\tilde{x}_{i} \neq \tilde{y}_{i}, \\
\mathscr{P}\left\{\omega:\left(\tilde{x}_{i}-\tilde{y}_{i}\right)\left(F_{i}(\tilde{x}, \omega)-F_{i}(\tilde{y}, \omega)\right)>0(\geq 0)\right\}=0 .
\end{gathered}
$$

On the other hand, since $F(\cdot, \bar{\omega})$ is a $P\left(P_{0}\right)$-function, then for $\tilde{x}, \tilde{y}$ there exist $i \in J(\tilde{x}, \tilde{y}), i \in\langle 1, n\rangle$ such that

$$
\left(\tilde{x}_{i}-\tilde{y}_{i}\right)\left(F_{i}(\tilde{x}, \bar{\omega})-F_{i}(\tilde{y}, \bar{\omega})\right)>0(\geq 0) .
$$

Notice that $\bar{\omega} \in \operatorname{supp} \Omega$, by the definition of $\operatorname{supp} \Omega$ in (16), we have

$$
\mathscr{P}\left\{\bar{\omega}:\left(\tilde{x}_{i}-\tilde{y}_{i}\right)\left(F_{i}(\tilde{x}, \bar{\omega})-F_{i}(\tilde{y}, \bar{\omega})\right)>0(\geq 0)\right\}>0 .
$$

This contradicts formulation (22). Therefore, $F$ is a stochastic $P\left(P_{0}\right)$-function.

Now for the "only if" part, suppose on the contrary that there does not exist a $\bar{\omega} \in \operatorname{supp} \Omega$ such that $F(\cdot, \bar{\omega})$ is a $P\left(P_{0}\right)$ function. Then for any $i \in\langle 1, n\rangle, \omega \in \operatorname{supp} \Omega$, there exists $\bar{x}, \bar{y}, \bar{x} \neq \bar{y}$ in $\mathbf{R}^{n}$, such that

$$
\begin{gathered}
\bar{x}_{i} \neq \bar{y}_{i}, \\
\left(\bar{x}_{i}-\bar{y}_{i}\right)\left(F_{i}(\bar{x}, \omega)-F_{i}(\bar{y}, \omega)\right) \leq 0(<0),
\end{gathered}
$$

which means that

$$
\begin{gathered}
\bar{x}_{i} \neq \bar{y}_{i}, \\
\mathscr{P}\left\{\omega \in \operatorname{supp} \Omega:\left(\bar{x}_{i}-\bar{y}_{i}\right)\left(F_{i}(\bar{x}, \omega)-F_{i}(\bar{y}, \omega)\right)\right. \\
>0(\geq 0)\}=0 .
\end{gathered}
$$

By the definition of supp $\Omega$ in (16), we have $\mathscr{P}\{\omega \in \Omega \backslash$ $\operatorname{supp} \Omega\}=0$. Hence, formulation (26) is equivalent to

$$
\bar{x}_{i} \neq \bar{y}_{i},
$$

$$
\mathscr{P}\left\{\omega \in \Omega:\left(\bar{x}_{i}-\bar{y}_{i}\right)\left(F_{i}(\bar{x}, \omega)-F_{i}(\bar{y}, \omega)\right)>0(\geq 0)\right\}=0,
$$

which contradicts definition (20). Therefore, there exists a $\bar{\omega} \in \operatorname{supp} \Omega$ such that $F(\cdot, \bar{\omega})$ is a $P\left(P_{0}\right)$-function.

Theorem 8. Suppose that $f(x):=\mathbf{E}[F(x, \omega)]$ is a $P\left(P_{0}\right)$ function. Then $F$ is a stochastic $P\left(P_{0}\right)$-function.

Proof. Suppose on the contrary that $F$ is not a stochastic $P\left(P_{0}\right)$-function, then there exist $\tilde{x}, \tilde{y}, \tilde{x} \neq \tilde{y}$ in $\mathbf{R}^{n}$ for any $i \epsilon$ $\langle 1, n\rangle$ satisfying

$$
\begin{gathered}
\tilde{x}_{i} \neq \tilde{y}_{i}, \\
\mathscr{P}\left\{\omega:\left(\tilde{x}_{i}-\tilde{y}_{i}\right)\left(F_{i}(\tilde{x}, \omega)-F_{i}(\tilde{y}, \omega)\right)>0(\geq 0)\right\}=0 .
\end{gathered}
$$

This means that

$$
\left(\tilde{x}_{i}-\tilde{y}_{i}\right)\left(F_{i}(\tilde{x}, \omega)-F_{i}(\tilde{y}, \omega)\right) \leq 0(<0)
$$


always holds for any $i \in\langle 1, n\rangle$ and $\omega \in \Omega$. Furthermore, following from (29), we have

$$
\mathbf{E}\left[\left(\tilde{x}_{i}-\tilde{y}_{i}\right)\left(F_{i}(\tilde{x}, \omega)-F_{i}(\tilde{y}, \omega)\right)\right] \leq 0(<0),
$$

that is

$$
\left(\tilde{x}_{i}-\tilde{y}_{i}\right)\left(f_{i}(\tilde{x})-f_{i}(\tilde{y})\right) \leq 0(<0),
$$

which contradicts the definition of $P\left(P_{0}\right)$-function. Therefore, $F$ is a stochastic $P\left(P_{0}\right)$-function.

Note that there is at most one solution (may not be a solution) for the EV model stochastic complementarity problems if $f(x):=\mathbf{E}[F(x, \omega)]$ is a $P\left(P_{0}\right)$-function.

\section{Boundedness of Solution Set}

Theorem 9. Suppose that $F$ is a stochastic uniformly $P$ function and $F$ is equicoercive on $\mathbf{R}^{n}$. Then the solution set of ERM model (7) defined by $\phi_{\min }$ and $\phi_{F B}$ is nonempty and bounded.

Proof. Suppose on the contrary that the ERM model defined by $\phi_{\text {min }}$ is not bounded. Thus there exist a sequence $\left\{x^{k}\right\} \subset \mathbf{R}_{+}^{n}$ with $\left\|x^{k}\right\| \rightarrow \infty(k \rightarrow \infty)$ and a constant $c \in \mathbf{R}_{+}$, such that

$$
\theta\left(x^{k}\right) \leq c, \quad \text { for } \forall k \text {. }
$$

Define the index set $I \subseteq\{1, \ldots, n\}$ by

$$
I:=\left\{i \mid\left\{x_{i}^{k}\right\} \text { is unbounded }\right\} .
$$

By assumption, we have $I \neq \emptyset$. We now define a sequence $\left\{y^{k}\right\} \subseteq \mathbf{R}^{n}$ as follows:

$$
y_{i}^{k}:= \begin{cases}0 & \text { if } i \in I, \\ x_{i}^{k} & \text { if } i \notin I .\end{cases}
$$

From the definition of $y^{k}$ and the fact that $F$ is a stochastic uniformly $P$-function, we obtain that for any $x^{k}, y^{k}$, there exists $i$ such that

$$
\begin{gathered}
\mathscr{P}\left\{\omega:\left(x_{i}^{k}-y_{i}^{k}\right)\left(F_{i}\left(x^{k}, \omega\right)-F_{i}\left(y^{k}, \omega\right)\right)\right. \\
\left.\geq \alpha\left\|x^{k}-y^{k}\right\|^{2}\right\}>0,
\end{gathered}
$$

and hence there are $\bar{\omega}^{k} \in \operatorname{supp} \Omega$ satisfying

$$
\left(x_{i}^{k}-y_{i}^{k}\right)\left(F_{i}\left(x^{k}, \bar{\omega}^{k}\right)-F_{i}\left(y^{k}, \bar{\omega}^{k}\right)\right) \geq \alpha\left\|x^{k}-y^{k}\right\|^{2} .
$$

Take subsequence $x^{k_{i}}, y^{k_{i}}$ such that the corresponding subscript of (36) is $j$. Noting that $j \in I$ and taking (36) into account, we have

$$
\begin{aligned}
& \alpha \sum_{j \in I}\left(x_{j}^{k_{i}}\right)^{2} \\
& \quad \leq x_{j}^{k_{i}}\left(F_{j}\left(x^{k_{i}}, \bar{\omega}^{k_{i}}\right)-F_{j}\left(y^{k_{i}}, \bar{\omega}^{k_{i}}\right)\right) \\
& \quad \leq \sqrt{\sum_{j \in I}\left(x_{j}^{k_{i}}\right)^{2}} \cdot \sqrt{\sum_{j \in I}\left|F_{j}\left(x^{k_{i}}, \bar{\omega}^{k_{i}}\right)-F_{j}\left(y^{k_{i}}, \bar{\omega}^{k_{i}}\right)\right|},
\end{aligned}
$$

from which we get

$$
\alpha \sqrt{\sum_{j \in I}\left(x_{j}^{k_{i}}\right)^{2}} \leq \sqrt{\sum_{j \in I}\left|F_{j}\left(x^{k_{i}}, \bar{\omega}^{k_{i}}\right)-F_{j}\left(y^{k_{i}}, \bar{\omega}^{k_{i}}\right)\right|} .
$$

By definition, the sequence $\left\{y^{k_{i}}\right\}$ remains bounded. From the continuity of $F$, it follows that the sequence $\left\{F_{j}\left(y^{k_{i}}, \omega^{k_{i}}\right)\right\}$ is also bounded for every $j \in I$. Hence, taking a limit in (38), we obtain that there is at least one index $\bar{j} \in I$ such that

$$
x_{\bar{j}}^{k_{i}} \longrightarrow \infty, \quad F_{\bar{j}}\left(x^{k_{i}}, \bar{\omega}^{k_{i}}\right) \longrightarrow \infty .
$$

Since $F$ is equicoercive on $\mathbf{R}^{n}$, we have

$$
\mathscr{P}\left\{\omega: \lim _{k_{i} \rightarrow \infty} F_{\bar{j}}\left(x^{k_{i}}, \omega\right)=\infty\right\}>0 .
$$

Let

$$
\Omega_{1}=\left\{\omega: \lim _{k_{i} \rightarrow \infty} \min \left(x_{\bar{j}}^{k_{i}}, F_{\bar{j}}\left(x^{k_{i}}, \omega\right)\right)=\infty\right\} .
$$

Then $\mathscr{P}\left\{\Omega_{1}\right\}>0$. By Fatou's Lemma [15], we have

$$
\begin{aligned}
\mathbf{E}_{\Omega_{1}} & {\left[\liminf _{k_{i} \rightarrow \infty}\left(\min \left(x_{\bar{j}}^{k_{i}}, F_{\bar{j}}\left(x^{k_{i}}, \omega\right)\right)\right)^{2}\right] } \\
& \leq \liminf _{k_{i} \rightarrow \infty} \mathbf{E}_{\Omega_{1}}\left[\left(\min \left(x_{\bar{j}}^{k_{i}}, F_{\bar{j}}\left(x^{k_{i}}, \omega\right)\right)\right)^{2}\right] .
\end{aligned}
$$

Since

$$
\liminf _{k_{i} \rightarrow \infty}\left(\min \left(x_{\bar{j}}^{k_{i}}, F_{\bar{j}}\left(x^{k_{i}}, \omega\right)\right)\right)^{2}=\infty
$$

on $\Omega_{1}$ and $\mathscr{P}\left\{\Omega_{1}\right\}>0$, then the left-hand side of formulation (42) is infinite. Therefore,

$$
\liminf _{k_{i} \rightarrow \infty} \mathbf{E}_{\Omega_{1}}\left[\left(\min \left(x_{\bar{j}}^{k_{i}}, F_{\bar{j}}\left(x^{k_{i}}, \omega\right)\right)\right)^{2}\right]=\infty .
$$

Moreover, it is easy to find

$$
\begin{aligned}
\theta\left(x^{k_{i}}\right) & =\mathbf{E}\left[\left\|\Phi\left(x^{k_{i}}, F\left(x^{k_{i}}, \omega\right)\right)\right\|^{2}\right] \\
& \geq \mathbf{E}_{\Omega_{1}}\left[\left(\min \left(x_{\bar{j}}^{k_{i}}, F_{\bar{j}}\left(x^{k_{i}}, \omega\right)\right)\right)^{2}\right] \\
& \longrightarrow \infty
\end{aligned}
$$

as $k_{i} \rightarrow \infty$. This contradicts formulation (32). Hence, the solution set of ERM model (7) defined by $\phi_{\min }$ is nonempty and bounded. Similar results about $\phi_{\mathrm{FB}}$ can be obtained by relation formulation (10).

\section{Robust Solution}

As we show, both EV model and ERM model give decisions by a deterministic formulation. However, the decisions may not be the best or may be even infeasible for each individual event. In fact, we should take risk into account to make 
a priori decision in many cases. Naturally, it is necessary to know how good or how bad the decision which we have given can be. In this section, we study the robustness of solutions of the ERM model. Let $\operatorname{SOL}(F(x, \omega))$ denote the solution set of $\operatorname{SCP}(F(x, \omega))$, and define the distance from a point $x$ to the set $\operatorname{SOL}(F(x, \omega))$ by

$$
\operatorname{dist}(x, \operatorname{SOL}(F(x, \omega))):=\inf _{x^{\prime} \in \operatorname{SOL}(F(x, \omega))}\left\|x-x^{\prime}\right\| .
$$

Theorem 10. Assume that $\Omega=\left\{\omega^{1}, \omega^{2}, \ldots, \omega^{N}\right\} \subset \mathbf{R}^{m}$, and $\omega$ takes values $\omega^{1}, \ldots, \omega^{N}$ with respective probabilities $p_{1}, \ldots, p_{N}$. Furthermore, suppose that for every $\omega \in \Omega, F(x, \omega)$ is uniformly P-function and Lipschitz continuous with respect to $x$. Then there is a constant $C>0$ such that

$$
\mathbf{E}[\operatorname{dist}(x, \operatorname{SOL}(F(x, \omega)))] \leq C \cdot \sqrt{\theta(x)},
$$

where $\theta(x)$ is defined by $\phi_{\min }$ or $\phi_{F B}$.

Proof. For any fixed $\omega^{i}$, since $F\left(x, \omega^{i}\right)$ is uniformly $P$ function and Lipschitz continuous, from Corollary 3.19 of [16], we have unique solution $\widehat{x}\left(\omega^{i}\right)$ of $\mathrm{CP}\left(F\left(x, \omega^{i}\right)\right)$, and there exists a constant $C_{i}$ such that

$$
\left\|x-\widehat{x}\left(\omega^{i}\right)\right\| \leq C_{i}\left\|\min \left\{x, F\left(x, \omega^{i}\right)\right\}\right\| .
$$

Letting $C:=((\sqrt{2}+2) / 2) \max \left\{C_{1}, \ldots, C_{N}\right\}$, we have

$$
\begin{aligned}
\mathbf{E}^{2}[ & \operatorname{dist}(x, \operatorname{SOL}(F(x, \omega)))] \\
= & \mathbf{E}^{2}\left[\left\|x-\widehat{x}\left(\omega^{i}\right)\right\|\right] \\
\leq & \mathbf{E}\left[\left\|x-\widehat{x}\left(\omega^{i}\right)\right\|^{2}\right] \\
\leq & \sum_{i=1}^{N} p_{i} \cdot C_{i}^{2}\left\|\min \left\{x, F\left(x, \omega^{i}\right)\right\}\right\|^{2} \\
\leq & \sum_{i=1}^{N} p_{i} \cdot C_{i}^{2} \cdot\left(\frac{\sqrt{2}+2}{2}\right)^{2} \\
& \times \sum_{j=1}^{n}\left(\sqrt{F_{j}^{2}\left(x, \omega^{i}\right)+x_{j}^{2}}-\left(F_{j}\left(x, \omega^{i}\right)+x_{j}\right)\right)^{2} \\
\leq & C^{2} \sum_{i=1}^{N} p_{i} \sum_{j=1}^{n}\left(\sqrt{F_{j}^{2}\left(x, \omega^{i}\right)+x_{j}^{2}}-\left(F_{j}\left(x, \omega^{i}\right)+x_{j}\right)\right)^{2} \\
= & C^{2} \theta(x),
\end{aligned}
$$

where the first inequality follows from Cauchy-Schwarz inequality, the second inequality follows from formulation (48), and the third inequality follows from formulation (10). This completes the proof of the theorem. (7),

Theorem 10 particularly shows that for the solution $x^{*}$ of

$$
\mathbf{E}\left[\operatorname{dist}\left(x^{*}, \operatorname{SOL}(F(x, \omega))\right)\right] \leq C \cdot \sqrt{\theta\left(x^{*}\right)} .
$$

This inequality indicates that the expected distance to the solution set $\operatorname{SOL}(F(x, \omega))$ for $\omega \in \Omega$ is also likely to be small at the solution $x^{*}$ of (7). In other words, we may expect that a solution of the ERM formulation (7) has a minimum sensitivity with respect to random parameter variations in $\operatorname{SCP}(F(x, \omega))$. In this sense, solutions of (7) can be regarded as robust solutions for $\operatorname{SCP}(F(x, \omega))$.

\section{Quasi-Monte Carlo and Derivative-Free Methods for Solving ERM Model}

Note that the ERM model (7) included an expectation function, which is generally difficult to be evaluated exactly. Hence in this section, we first employ a quasi-Monte Carlo method to obtain approximation problems of (7) for numerical integration. Then, we consider derivative-free methods to solve these approximation problems.

By the quasi-Monte Carlo method, we obtain the following approximation problem of (7):

$$
\min _{x \in \mathbf{R}_{+}^{n}} \theta^{N}(x):=\frac{1}{N} \sum_{\omega^{i} \in \Omega_{N}}\left\|\Phi\left(x, \omega^{i}\right)\right\|^{2} \rho\left(\omega^{i}\right),
$$

where $\Omega_{N}=\left\{\omega^{i} \mid i=1,2, \ldots, N\right\}$ is a set of observations generated by a quasi-Monte Carlo method such that $\Omega_{N} \subseteq$ $\Omega$ and $\rho(\omega)$ stands for the probability density function. In the rest of this paper, we assume that the probability density function $\rho$ is continuous on $\Omega$. For each $N, \theta^{N}(x)$ is continuously differentiable function. We denote by $x^{N}$ the optimal solutions of approximation problems (51). We are interested in the situation where the first-order derivatives of $\theta^{N}(x)$ cannot be explicitly calculated or approximated.

Condition 1. Given a point $x_{0} \geq 0$, the level set

$$
L:=\left\{x \geq 0 \mid f(x) \leq f\left(x_{0}\right)\right\}
$$

is compact.

Condition 2. If $\left\{x_{k}^{N}\right\}$ and $\left\{y_{k}^{N}\right\}$ are sequences of points such that $x_{k}^{N} \geq 0, y_{k}^{N} \geq 0$ converging to some $\bar{x}^{N}$ and $I_{k}^{N} \subseteq$ $I\left(\bar{x}^{N}\right):=\left\{i \mid \bar{x}_{i}^{N}=0\right\}$ for all $k$, then

$$
\left\{\operatorname{dist}\left(T_{I_{k}^{N}}\left(x_{k}^{N}\right), T_{I_{k}^{N}}\left(y_{k}^{N}\right)\right)\right\} \longrightarrow 0,
$$

where $\operatorname{dist}\left(T_{1}, T_{2}\right)=\max _{d_{1} \in T_{1}:\left\|d_{1}\right\|=1}\left\{\min _{d_{2} \in T_{2}}\left\|d_{1}-d_{2}\right\|\right\}$ and $T_{I_{k}^{N}}(x):=\left\{d_{k}^{N} \in \mathbf{R}^{n} \mid d_{k, i}^{N} \geq 0, \forall i \in I_{k}^{N}\right\}$.

Condition 3. For every $\bar{x}^{N} \geq 0$ there exist scalars $\delta>0$, and $\eta>0$ such that

$$
\begin{array}{r}
\min _{z \geq 0}\|z-x\| \leq \eta \sum_{i=1}^{n} \max \left(-x_{i}, 0\right), \\
\forall x \in\left\{x \in \mathbf{R}^{n} \mid\|x-\bar{x}\| \leq \delta\right\} .
\end{array}
$$

Condition 4. Given $x_{k}^{N}$ and $\epsilon_{k}^{N}>0$, the set of search directions

$$
D_{k}^{N}=\left\{d_{k}^{N, j}, j=1, \ldots, r_{k}^{N}\right\}, \quad \text { with }\left\|d_{k}^{N, j}\right\|=1,
$$


satisfing $r_{k}^{N}$ is uniformly bounded and cone $\left\{D_{k}^{N}\right\}=T\left(x_{k}^{N}\right.$; $\left.\epsilon_{k}^{N}\right)$. Here,

$$
\begin{aligned}
\text { cone } & \left\{D_{k}^{N}\right\} \\
= & \left\{d_{k}^{N, 1} \beta^{1}+\cdots+d_{k}^{N, r_{k}^{N}} \beta^{r_{k}^{N}}: \beta^{1} \geq 0, \ldots, \beta^{r_{k}^{N}} \geq 0\right\}, \\
& T\left(x_{k}^{N} ; \epsilon_{k}^{N}\right)=\left\{d_{k}^{N} \in \mathbf{R}^{n} \mid d_{k, i}^{N} \geq 0, x_{k, i}^{N} \leq \epsilon_{k}^{N}\right\} .
\end{aligned}
$$

Under Conditions 1, 2, and 3 and by choosing $D_{k}^{N}$ satisfying Condition 4 with $\epsilon_{k}^{N} \rightarrow 0$, then the following generated iterates have at least one cluster point that is a stationary point of (51) for each $N$.

Algorithm 11. Parameters: $x_{0}^{N} \geq 0, \widetilde{\alpha}_{0}^{N}>0, \gamma^{N}>0, \theta_{1}^{N} \in$ $(0,1), \theta_{2}^{N} \in(0,1), \epsilon_{0}^{N}>0$.

Step 1. Set $k^{N}=0$.

Step 2. Choose a set of directions $D_{k}^{N}=\left\{d_{k}^{N, j}, j=1, \ldots, r_{k}^{N}\right\}$ satisfying Condition 4.

Step 3 .

(a) Set $j=1, y_{k}^{N, j}=x_{k}^{N}, \widetilde{\alpha}_{k}^{N, j}=\widetilde{\alpha}_{k}^{N}$.

(b) Compute the maximum stepsize $\bar{\alpha}_{k}^{N, j}$ such that $y_{i, k}^{N, j}+$ $\bar{\alpha}_{k}^{N, j} \widehat{d}_{i, k}^{N, j} \geq 0$ for all $i$. Set $\widehat{\alpha}_{k}^{N, j}=\min \left\{\bar{\alpha}_{k}^{N, j}, \widetilde{\alpha}_{k}^{N, j}\right\}$.

(c) If $\widehat{\alpha}_{k}^{N, j}>0$ and $\theta^{N}\left(\widehat{y}_{k}^{N, j}\right) \leq \theta^{N}\left(y_{k}^{N, j}\right)-\gamma\left(\widehat{\alpha}_{k}^{N, j}\right)^{2}$, set $\tilde{\alpha}_{k}^{N, j+1}=\alpha_{k}^{N, j}$; otherwise set $\alpha_{k}^{N, j}=0, y_{k}^{N, j+1}=$ $y_{k}^{N, j}, \widetilde{\alpha}_{k}^{N, j+1}=\theta_{1}^{N} \widetilde{\alpha}_{k}^{N, j}$.

(d) If $\alpha_{k}^{N, j}=\bar{\alpha}_{k}^{N, j}$, set $\epsilon_{k+1}^{N}=\epsilon_{k}^{N}$, and go to Step 4 .

(e) If $j<r_{k}^{N}$, set $j=j+1$, and go to Step 3(b). Otherwise set $\epsilon_{k+1}^{N}=\theta_{2}^{N} \epsilon_{k}^{N}$ and go to Step 4 .

Step 4. Find $x_{k+1}^{N} \geq 0$ such that $\theta^{N}\left(x_{k+1}^{N}\right) \leq \theta^{N}\left(y_{k}^{N, j+1}\right)$. Set $\widetilde{\alpha}_{k+1}^{N}=\widetilde{\alpha}_{k}^{N, j+1}, \widetilde{r}_{k}=j, k=k+1$, and go to Step 2 .

For this algorithm, it is easy to proof that if $x_{k}^{N}$ is the sequence produced by algorithm under Conditions $1-4$, then $x_{k}^{N}$ is bounded and there exists at least one cluster point which is a stationary point of problem (51) for each $N$.

\section{Conclusions}

The $\operatorname{SCP}(F(x, \omega))$ has a wide range of applications in engineering and economics. Therefore, it is meaningful and interesting to study this problem. In this paper, we give the definitions of stochastic $P$-function, stochastic $P_{0}$-function and stochastic uniformly $P$-function, which can be regarded as a generalization of the deterministic formulation or an extension of a stochastic $R_{0}$ function given in [11]. Moreover, we consider the conditions when the function is a stochastic $P\left(P_{0}\right)$-function. Furthermore, we show that the involved function being a stochastic uniformly $P$-function and equicoercive [11] are sufficient conditions for the solution set of the expected residual minimization problem to be nonempty and bounded. Finally, we illustrate that the ERM formulation produces robust solutions with minimum sensitivity in violation of feasibility with respect to random parameter variations in $\operatorname{SCP}(F(x, \omega))$. On the other hand, we employ a quasiMonte Carlo method to obtain approximation problems of (7) for dealing numerical integration and further consider derivative-free methods to solve these approximation problems.

\section{Acknowledgments}

This work was supported by NSFC Grants no. 11226238 and no. 11226230, and predeclaration fund of state project of Liaoning university 2012, 2012LDGY01, and University Scientific Research Projects of School of Education Department of Liaoning Province 2012, 2012427.

\section{References}

[1] F. Facchinei and J. S. Pang, Finite-Dimensional Variational Inequalities and Complementarity Problems, Springer, New York, NY, USA, 2003.

[2] R. W. Cottle, J.-S. Pang, and R. E. Stone, The Linear Complementarity Problem, Computer Science and Scientific Computing, Academic Press, Boston, Mass, USA, 1992.

[3] G.-H. Lin and M. Fukushima, "New reformulations for stochastic nonlinear complementarity problems," Optimization Methods \& Software, vol. 21, no. 4, pp. 551-564, 2006.

[4] G. Gürkan, A. Y. Özge, and S. M. Robinson, "Sample-path solution of stochastic variational inequalities," Mathematical Programming, vol. 84, no. 2, pp. 313-333, 1999.

[5] X. Chen and M. Fukushima, "Expected residual minimization method for stochastic linear complementarity problems," Mathematics of Operations Research, vol. 30, no. 4, pp. 1022-1038, 2005.

[6] X. Chen, C. Zhang, and M. Fukushima, "Robust solution of monotone stochastic linear complementarity problems," Mathematical Programming, vol. 117, no. 1-2, pp. 51-80, 2009.

[7] P. Tseng, "Growth behavior of a class of merit functions for the nonlinear complementarity problem," Journal of Optimization Theory and Applications, vol. 89, no. 1, pp. 17-37, 1996.

[8] H. Fang, X. Chen, and M. Fukushima, "Stochastic $R_{0}$ matrix linear complementarity problems," SIAM Journal on Optimization, vol. 18, no. 2, pp. 482-506, 2007.

[9] G.-H. Lin, X. Chen, and M. Fukushima, "New restricted NCP functions and their applications to stochastic NCP and stochastic MPEC," Optimization, vol. 56, no. 5-6, pp. 641-953, 2007.

[10] C. Ling, L. Qi, G. Zhou, and L. Caccetta, “The $S C^{1}$ property of an expected residual function arising from stochastic complementarity problems," Operations Research Letters, vol. 36, no. 4, pp. 456-460, 2008.

[11] C. Zhang and X. Chen, "Stochastic nonlinear complementarity problem and applications to traffic equilibrium under uncertainty," Journal of Optimization Theory and Applications, vol. 137, no. 2, pp. 277-295, 2008. 
[12] C. Zhang and X. Chen, "Smoothing projected gradient method and its application to stochastic linear complementarity problems," SIAM Journal on Optimization, vol. 20, no. 2, pp. 627-649, 2009.

[13] G. L. Zhou and L. Caccetta, "Feasible semismooth Newton method for a class of stochastic linear complementarity problems," Journal of Optimization Theory and Applications, vol. 139, no. 2, pp. 379-392, 2008.

[14] X. L. Li, H. W. Liu, and Y. K. Huang, "Stochastic $P$ matrix and $P_{0}$ matrix linear complementarity problem," Journal of Systems Science and Mathematical Sciences, vol. 31, no. 1, pp. 123-128, 2011.

[15] K. L. Chung, A Course in Probability Theory, Academic Press, New York, NY, USA, 2nd edition, 1974.

[16] B. Chen and P. T. Harker, "Smooth approximations to nonlinear complementarity problems," SIAM Journal on Optimization, vol. 7, no. 2, pp. 403-420, 1997. 


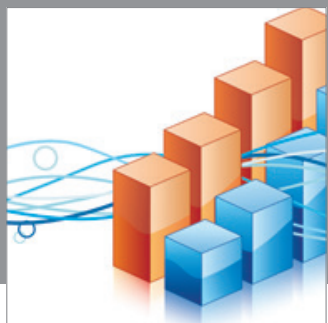

Advances in

Operations Research

mansans

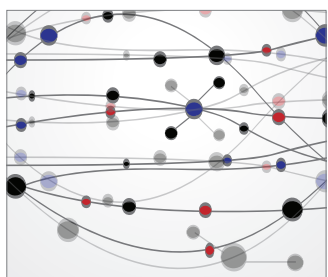

The Scientific World Journal
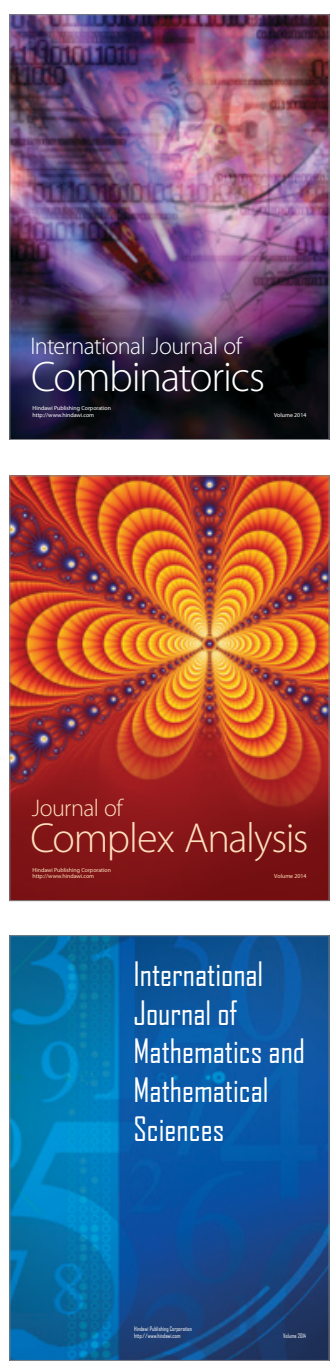
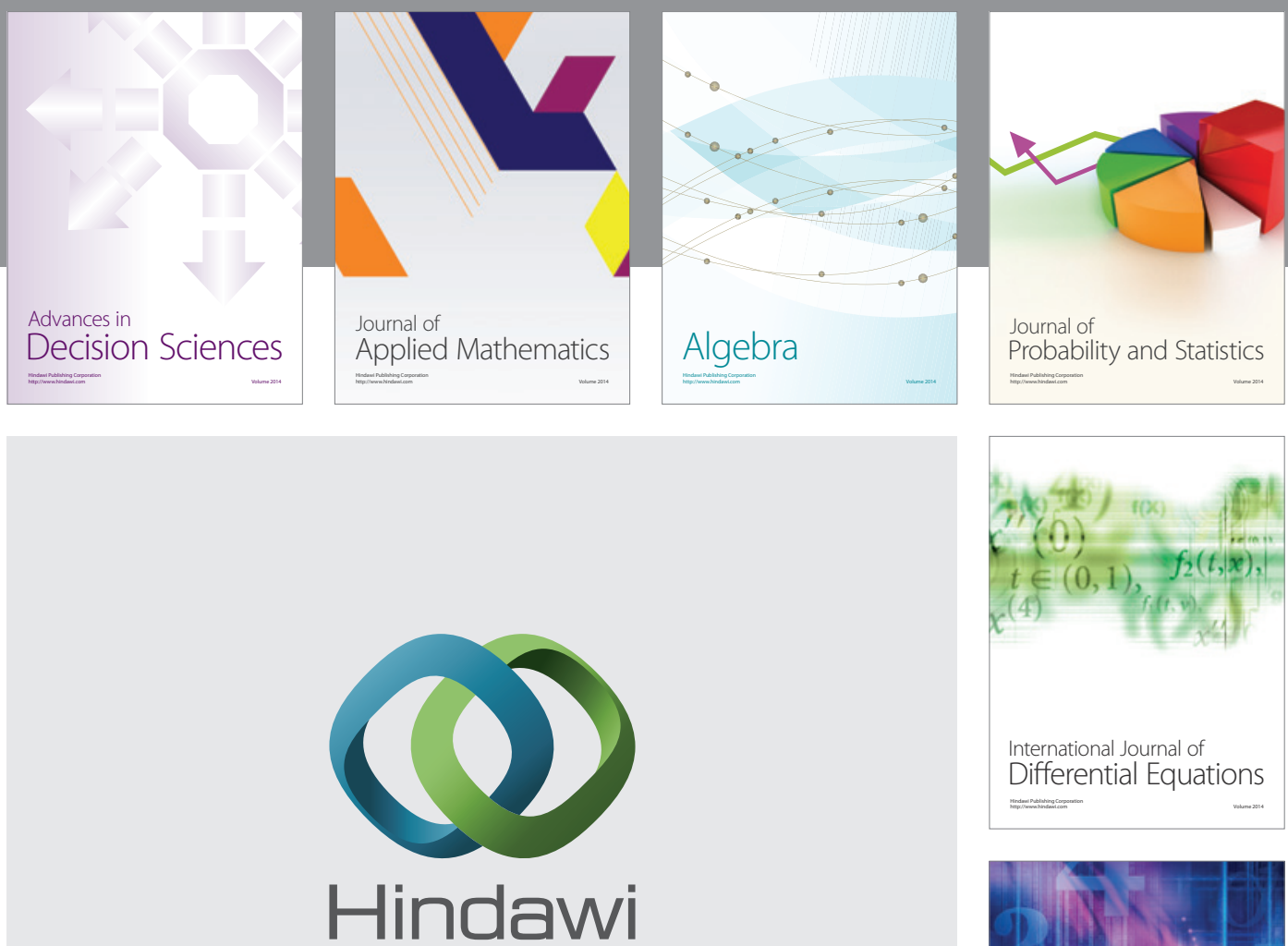

Submit your manuscripts at http://www.hindawi.com
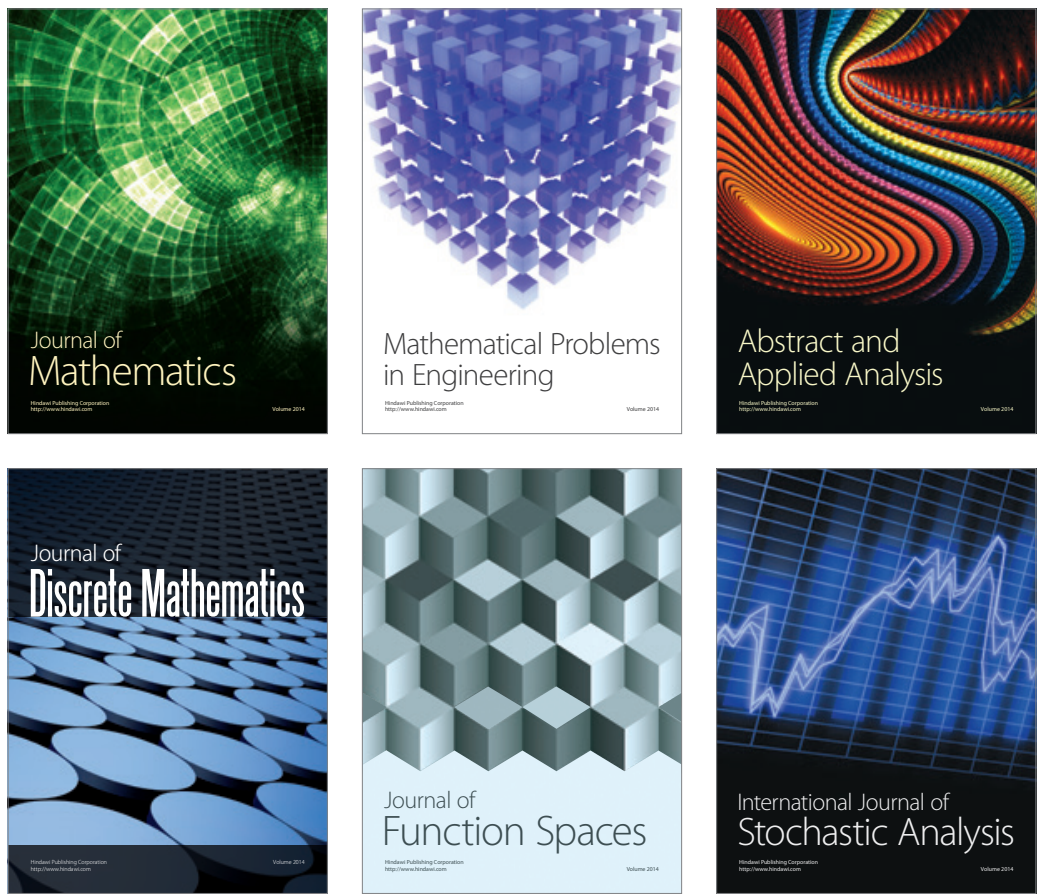

Journal of

Function Spaces

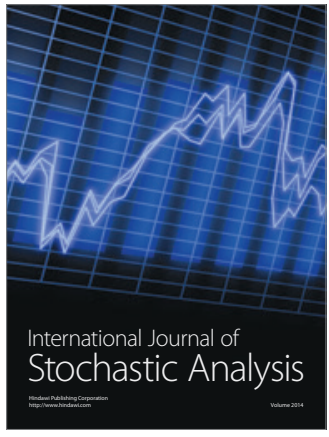

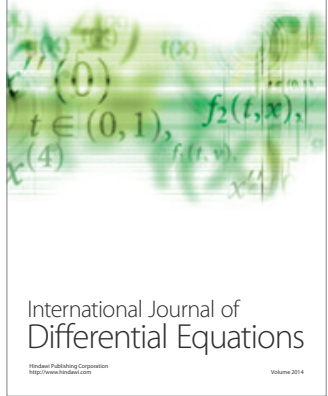
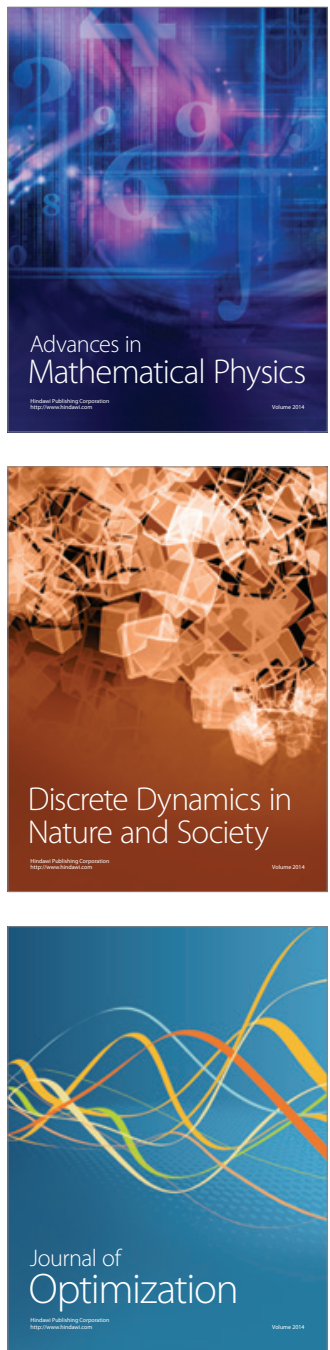